Draft VERSion MAY 31, 2018

Preprint typeset using $\mathrm{L}^{A} \mathrm{~T}_{\mathrm{E}} \mathrm{X}$ style emulateapj v. 11/10/09

\title{
THE SPECTRAL ENERGY DISTRIBUTION OF POST-STARBURST GALAXIES IN THE NEWFIRM MEDIUM-BAND SURVEY: A LOW CONTRIBUTION FROM TP-AGB STARS
}

\author{
Mariska Kriek $^{1}$, Ivo Labbé ${ }^{2}$, Charlie Conroy ${ }^{1,3}$, Katherine E. Whitaker ${ }^{4}$, Pieter G. van Dokkum ${ }^{4}$, Gabriel B. \\ Brammer $^{4}$, Marijn Franx ${ }^{5}$, Garth D. Illingworth ${ }^{6}$, Danilo Marchesini $^{7}$, Adam Muzzin $^{4}$, Ryan F. Quadri $^{5}, \&$ \\ GREGORY RUDNICK ${ }^{8}$ \\ Draft version May 31, 2018
}

\begin{abstract}
Stellar population synthesis (SPS) models are a key ingredient of many galaxy evolution studies. Unfortunately, the models are still poorly calibrated for certain stellar evolution stages. Of particular concern is the treatment of the thermally-pulsing asymptotic giant branch (TP-AGB) phase, as different implementations lead to systematic differences in derived galaxy properties. Post-starburst galaxies are a promising calibration sample, as TP-AGB stars are thought to be most prominently visible during this phase. Here, we use post-starburst galaxies in the NEWFIRM medium-band survey to assess different SPS models. The available photometry allows the selection of a homogeneous and well-defined sample of 62 post-starburst galaxies at $0.7 \lesssim z \lesssim 2.0$, from which we construct a well-sampled composite spectral energy distribution (SED) over the range $1200-40000 \AA$. The SED is well fit by the Bruzual \& Charlot SPS models, while the Maraston models do not reproduce the rest-frame optical and near-infrared parts of the SED simultaneously. When the fitting is restricted to $\lambda<6000 \AA$, the Maraston models overpredict the near-infrared luminosity, implying that these models give too much weight to TP-AGB stars. Using the flexible SPS models by Conroy et al, and assuming solar metallicity, we find that the contribution of TP-AGB stars to the integrated SED is a factor of $\sim 3$ lower than predicted by the latest Padova TP-AGB models. Whether this is due to lower bolometric luminosities, shorter lifetimes, and/or heavy dust obscuration of TP-AGB stars remains to be addressed. Altogether, our data demand a low contribution from TP-AGB stars to the SED of post-starburst galaxies.
\end{abstract}

Subject headings: galaxies: evolution — galaxies: stellar content — stars: AGB and post-AGB

\section{INTRODUCTION}

Galaxy evolution studies at all epochs strongly rely on stellar population synthesis (SPS) models (e.g., Tinsley \& Gunn 1976; Leitherer et al. 1999; Bruzual \& Charlot 2003; Maraston 2005; Conroy et al. 2009). These models are key in deriving stellar masses, stellar population properties, and in some cases even redshifts from spectra and broadband photometry. Thus, our current understanding of stellar populations and galaxy growth across cosmic time (e.g., Papovich et al. 2001; Förster Schreiber et al. 2004; Shaplev et al. 2005; Kriek et al. 2009a; van Dokkum et al. 2010), and the evolution of the stellar mass density (e.g. Rudnick et al. 2006; Stark et al. 2007; Marchesini et al. 2009; Gonzalez et al. 2010; Labbé et al. 2010) is critically dependent on SPS models.

\footnotetext{
${ }^{1}$ Department of Astrophysical Sciences, Princeton University, Princeton, NJ 08544, USA

${ }^{2}$ Carnegie Observatories, 813 Santa Barbara Street, Pasadena, CA 91101, USA

${ }^{3}$ Harvard-Smithsonian Center for Astrophysics, 60 Garden Street, Cambridge, MA 02138, USA

${ }_{4}^{4}$ Department of Astronomy, Yale University, New Haven, CT 06520, USA

${ }^{5}$ Leiden Observatory, Leiden University, NL-2300 RA Leiden, The Netherlands

${ }^{6} \mathrm{UCO} /$ Lick Observatory, University of California, Santa Cruz, CA 95064, USA

${ }^{7}$ Department of Physics and Astronomy, Tufts University, Medford, MA 02155, USA

${ }^{8}$ Department of Physics and Astronomy, The University of Kansas, Lawrence, KS 66045, USA
}

Nonetheless, SPS models are still poorly calibrated for certain stellar evolution stages. In particular, the thermally pulsing asymptotic giant branch (TP-AGB) phase is a source of major discrepancy among different SPS models; different implementations of its treatment lead to large systematic differences in derived galaxy properties. TP-AGB stars are cool giants $(T \lesssim 4000 \mathrm{~K})$ and primarily contribute to the near-infrared part of the spectrum. They are of low-to-intermediate stellar mass, and thus their relative contribution to the near-infrared spectrum is highest around 0.5-2.0 Gyr after a starburst. Due to the short lifetime of AGB stars ( $1 \mathrm{Myr})$, the local globular cluster calibration samples are small, and the total luminosity, temperature, and carbon star fraction in the TP-AGB phase are not well calibrated. For example, the TP-AGB phase is more prominent in the Maraston (2005) models than in Bruzual \& Charlot (2003). Therefore, stellar masses derived using the Bruzual \& Charlot (2003) models are generally higher than stellar masses derived using the Maraston (2005) SPS models (e.g., Wuvts et al.|2007; Muzzin et al.|2009; Kannappan \& Gawiser 2007; van der Wel et al. 2006).

Several studies use galaxy spectral energy distributions (SEDs) to assess the different SPS models, and resolve the discrepancies (e.g, Maraston et al. 2006; Eminian et al. 2008). This exercise is complicated due to the large variety of possible stellar populations and the degeneracies between the star formation timescale, age, metallicity, and dust. However, specific galaxy populations can be used to constrain certain stellar evolu- 


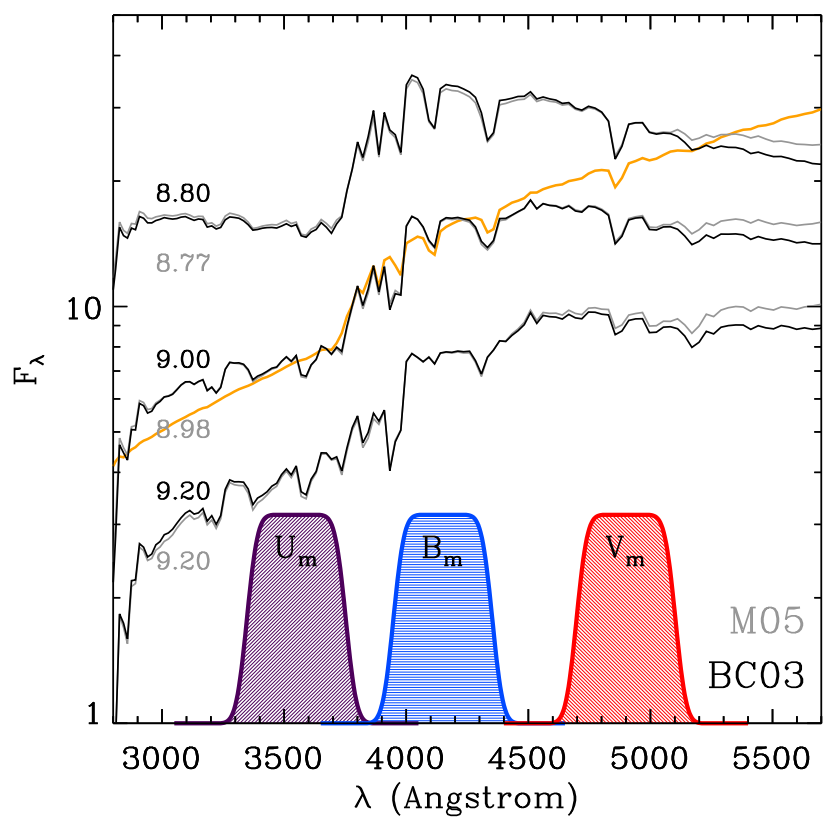

FIG. 1.- Synthetic filter curves of intermediate bandwidth used to select post-starburst galaxies. $U_{\mathrm{m}}-B_{\mathrm{m}}$ isolates the Balmer break, while $B_{\mathrm{m}}-V_{\mathrm{m}}$ measures the slope redwards of the break. The Bruzual \& Charlot (2003) and Maraston (2005) models for the same IMF (Salpeter 1955) are shown for ages of $\log (t / \mathrm{yr}) \approx 8.8,9.0$, and 9.2 , and an e-folding time of $\log (\tau / \mathrm{yr})=8.0$. We also show an SED of a dusty star-forming galaxy $(\tau=1 \mathrm{Gyr}$ and $t=100 \mathrm{Myr})$ with the same $U_{\mathrm{m}}-B_{\mathrm{m}}$ color as the $1 \mathrm{Gyr}$ old post-starburst galaxy (orange). All three filters fall in the regime where the model colors of post-starburst galaxies are nearly identical, and thus our selection is not biased toward any particular SPS model.

tion phases. For example, Conroy \& Gunn (2010) use post-starburst galaxies in the Sloan Digital Sky Survey (SDSS) to assess the treatment of the TP-AGB phase of different SPS models, as TP-AGB stars dominate the near-infrared luminosity during this phase. They find that the models by Bruzual \& Charlot (2003) and Conrov et al. (2009) can reproduce the optical and near-infrared colors of post-starburst galaxies, while the Maraston (2005) models cannot.

In this Letter, we take a similar approach as Conroy \& Gunn (2010) and use a post-starburst galaxy population in the NEWFIRM medium-band survey (NMBS; van Dokkum et al. 2009) to test different SPS models, and obtain new constraints on the SED shape during the time that the TP-AGB stars are thought to be most dominant. The NMBS allows us to photometrically select a large and clean sample of post-starburst galaxies, and to construct a composite SED which is more thoroughly sampled than in Conroy \& Gunn (2010). Throughout the Letter, we assume a $\Lambda$ CDM cosmology with $\Omega_{\mathrm{m}}=0.3, \Omega_{\Lambda}=0.7$, and $H_{0}=70 \mathrm{~km} \mathrm{~s}^{-1} \mathrm{Mpc}^{-1}$.

\section{SAMPLE SELECTION}

The NMBS uses five custom near-infrared mediumband filters and covers a total area of $0.4 \mathrm{deg}^{2}$ in the COSMOS (Scoville et al. 2007) and AEGIS (Davis et al. 2007) fields. The medium-band filter set, in combination with deep optical medium and broadband photometry and IRAC imaging, provides accurate photometric redshifts and stellar population properties

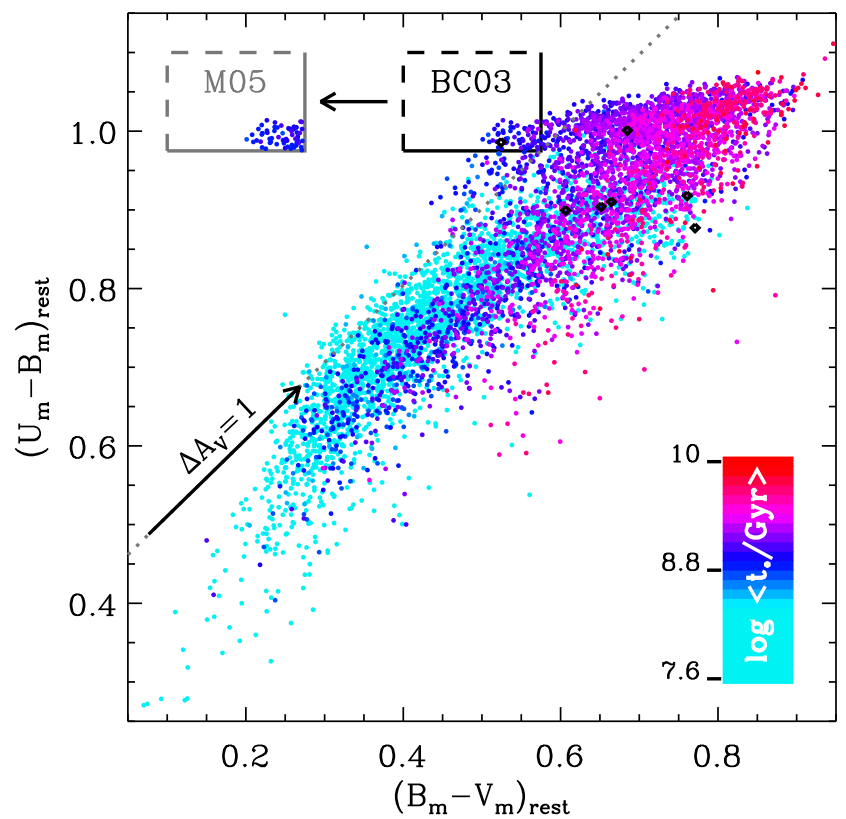

FIG. 2.- Rest-frame $U_{\mathrm{m}}-B_{\mathrm{m}}$ vs. $B_{\mathrm{m}}-V_{\mathrm{m}}$ for all $z>0.5$ galaxies in the NMBS (with $\left.(\mathrm{S} / \mathrm{N})_{K}>25\right)$. The black box indicates our post-starburst selection, characterized by red $U_{\mathrm{m}}-B_{\mathrm{m}}$ colors and relatively blue $B_{\mathrm{m}}-V_{\mathrm{m}}$ colors. The dust vector and dotted line indicate that contamination by obscured star-forming galaxies is minimal. The galaxies are color coded following their Bruzual \& Charlot (2003) average stellar ages. In the gray box, we show the Maraston (2005) average stellar ages of the selected galaxies. For both models the rest-frame near-infrared is downweighted when fitting. The black diamonds are the seven galaxies in Maraston et al. (2006).

(e.g., Brammer et al. 2009; van Dokkum et al. 2010; Whitaker et al. 2010). A full overview of this survey is given in K. E. Whitaker et al. (2010, in preparation). The photometric redshifts and stellar population properties are derived using EAZY (Brammer et al. 2008) and FAST (Kriek et al. 2009b), respectively.

Our post-starburst galaxy sample is selected using three optimized synthetic rest-frame filters of intermediate bandwidth $\left[9\right.$ (see Figure 10. The $U_{\mathrm{m}}$ and $B_{\mathrm{m}}$ filters isolate the Balmer break, while $B_{\mathrm{m}}-V_{\mathrm{m}}$ measures the slope of the SED just redwards of the Balmer break. The location of the $V_{\mathrm{m}}$ filter is a trade-off between being red enough to constrain the SED slope and blue enough not to enter the regime where the stellar evolution models and spectral libraries start to deviate (see also Maraston et al. 2009). Post-starburst galaxies have strong Balmer breaks, characterized by red $U_{\mathrm{m}}-B_{\mathrm{m}}$ colors and blue $B_{\mathrm{m}}-V_{\mathrm{m}}$ colors, and thus are expected to lie in the black selection box in Figure 2, The boundaries of the selection box are a trade-off between minimizing contamination by dusty star-forming galaxies (estimated to be $\sim 1 \%$ ) and obtaining a large enough sample. As indicated by the orange SED in Figure 1, dusty star-forming galaxies with the same $U_{\mathrm{m}}-B_{\mathrm{m}}$ color as post-starburst galaxies will have redder $B_{\mathrm{m}}-V_{\mathrm{m}}$ colors.

In order to solely include high-quality SEDs with restframe UV-to-NIR wavelength coverage, we require a red-

${ }^{9}$ Rest-frame colors are computed from the best-fit EAZY templates (see Brammer et al. 2009). 



FIG. 3.- Composite post-starburst SED (black dots), derived from the individual SEDs (gray dots) of 62 post-starburst galaxies in the NMBS. The individual SEDs are normalized at $5000 \AA$ before combining. The best-fit Bruzual \& Charlot (2003) and Maraston (2005) SPS models to the full spectrum, with the same resolution as the data, are shown by the orange and purple curves, respectively. The red and blue curves show the respective best fits when excluding $\lambda>6000 \AA$ in the fit. The inset represents the minimum $\chi^{2}$ value per degree of freedom vs. the mean stellar age for both SPS models. In the top panel, we show the fractional offset between the models and the data. The gray shaded area indicates the uncertainties on the composite SED. The Bruzual \& Charlot (2003) models more accurately reproduce the SED shape of post-starburst galaxies than the Maraston (2005) models.

shift of $z>0.5$ and a $K$-band signal-to-noise ratio $(\mathrm{S} / \mathrm{N})$ of 25 . We also exclude galaxies for which the IRAC fluxes are strongly contaminated by nearby sources, and thus cannot properly be deblended (Labbé et al. 2005). The final selection consists of 62 galaxies at $0.695<z<$ 2.028 , with a median redshift of $z=1.56$. Six targets have spectroscopic redshifts, yielding a median photometric redshift uncertainty of $\Delta z /(1+z)=0.005$.

We have inspected the SEDs of all targets by eye and none have obvious problems. Furthermore, for none of the selected galaxies there are indications that the restframe UV-to-NIR continuum is dominated by dusty star formation (Figure 22). Similar to spectroscopic poststarburst galaxy samples, obscured star formation may have been missed (e.g., Miller \& Owen 2001; Goto 2007). However, some contamination by a dusty star-forming population will not compromise our results (see Section $4.1)$.
To obtain a homogeneous sample and to avoid modeling degeneracies, our selection intentionally excludes younger or heavily dust-obscured post-starburst galaxies and specifically targets galaxies with short star formation timescales, for which the TP-AGB phase is most prominent. Therefore, our sample is likely more restrictive than other spectroscopically selected post-starburst galaxy samples (e.g., Quintero et al. 2004). However, for the purpose of this Letter it is not relevant whether our sample is complete, as long as the selection is not biased toward any particular SPS model.

\section{COMPOSITE SPECTRUM}

In order to make a composite spectrum, we deredshift the SEDs of all galaxies to rest frame, without changing the observed fluxes. All SEDs are normalized at $5000 \AA$, the longest wavelength for which the variations between the different SPS models are negligible (see Figure 11). 
TABLE 1

Stellar Population Properties

\begin{tabular}{|c|c|c|c|c|c|c|c|c|c|c|}
\hline ID & Fit region & Model & $\begin{array}{l}\log t \\
(\mathrm{yr})\end{array}$ & $\begin{array}{l}\log \tau \\
(\mathrm{yr})\end{array}$ & $\begin{array}{c}A_{V} \\
(\mathrm{mag})\end{array}$ & $Z$ & $\begin{array}{c}\log M_{*} \\
M_{\odot}\end{array}$ & $\begin{array}{l}\log \mathrm{SFR} \\
M_{\odot \mathrm{yr}^{-1}}\end{array}$ & $\underset{\mathrm{yr}^{-1}}{\log \mathrm{SFR} / M_{*}}$ & $\chi_{\text {red }}^{2}$ \\
\hline \multirow[t]{4}{*}{ Composite SED } & Full range & B & $9.06_{-0.01}^{+0.01}$ & $8.10_{-0.02}^{+0.04}$ & $0.16_{-0}^{+0}$ & 0.020 & $11.10_{-0.02}^{+0.00}$ & $-0.63_{-0.10}^{+0.01}$ & $-11.73_{-0.08}^{+0.00}$ & 0.73 \\
\hline & & M05 & $905+0$ & $8.10_{-0.00}^{+0.00}$ & $0.00_{-}^{+}$ & 0.020 & $10.99_{-0.00}^{+0.00}$ & $\begin{array}{l}-0.67_{-0.00}^{+0.00} \\
-\end{array}$ & $-11.66_{-0.00}^{+0.00}$ & 5.08 \\
\hline & $\lambda<6000 \AA$ & $\mathrm{BC} 03$ & $9.06_{-0.02}^{+0.18}$ & $8.10_{-0.05}^{+0.20}$ & $0.16_{-0.12}^{+0.15}$ & 0.020 & $11.10_{-0.05}^{+0.09}$ & $\begin{array}{l}-0.63_{-0.13}^{+0.00} \\
+0.18\end{array}$ & $-11.73_{-0.08}^{+0.14}$ & 0.72 \\
\hline & & M05 & $9.06_{-0.01}^{+0.01}$ & $8.10_{-0.03}^{+0.08}$ & $0.08_{-0.08}^{+0.08}$ & 0.020 & $11.09_{-0.12}^{+0.03}$ & $\begin{array}{r}-0.65_{-0.12}^{+0.13} \\
-10\end{array}$ & $\begin{array}{r}-11.74_{-0.08}^{+0.20} \\
\end{array}$ & 0.94 \\
\hline Ma-3650 & Full range & $\mathrm{BC} 03$ & $9.08_{-0.19}^{+0.01}$ & $8.10_{-1.10}^{+0.14}$ & $0.40_{-0.13}^{+0.45}$ & 0.008 & $10.89_{-0.00}^{+0.13}$ & $\begin{array}{l}-1.03_{-27.34}^{+0.47} \\
+0\end{array}$ & $\begin{array}{l}-11.92_{-27.45}^{+0.40} \\
-10\end{array}$ & 2.91 \\
\hline$z_{\mathrm{spec}}=1.91$ & & M05 & $8.94_{-0.17}^{+0.11}$ & $7.90_{-0.90}^{+0.22}$ & $0.10_{-0.10}^{+0.13}$ & 0.020 & $10.76_{-0.11}^{+0.03}$ & $-1.54_{-20.98}^{+0.73}$ & $\begin{array}{l}-12.30_{-20.97}^{+0.70} \\
\end{array}$ & 2.90 \\
\hline
\end{tabular}

Note. - We fit a grid with $\log (t / \mathrm{yr})$ between 7.6 and 10.1 , in steps of 0.01 (not exceeding the age of the universe), log $(\tau / \mathrm{yr})$ between 7 and 10 in steps of 0.1 , and $A_{V}$ between 0 and 3 in steps of 0.02 mag. For each model we explore three different metallicities: subsolar (0.01 and 0.008 for the Maraston (2005) and Bruzual \& Charlot (2003) models, respectively), solar, or supersolar (0.04 and 0.05 for the Maraston (2005) and Bruzual \& Charlot (2003) models, respectively). To account for systematic errors, we increase all photometric uncertainties by quadratically adding $5 \%$ of the photometric flux. The errors represent the $68 \%$ confidence intervals.

We create a composite SED by averaging the rest-frame fluxes in wavelength bins of 20 data points each. The uncertainties on the composite spectrum are determined by bootstrapping.

Figure 3 shows the composite post-starburst galaxy SED in black. The photometric data points of the individual galaxies are represented by the gray dots. The uniformity of all individual SEDs is remarkable and results in a high-quality, low-resolution spectrum of a poststarburst galaxy. Even subtle features, such as the Mg II absorption line at $2800 \AA$ and the continuum break at $2640 \AA$, are detected.

\section{COMPARISON TO MODELS}

\subsection{Bruzual \& Charlot (2003) and Maraston (2005)}

The high quality of the composite post-starburst galaxy SED allows the assessment of the different SPS models. We fit the composite SED by both the Bruzual \& Charlot (2003) and Maraston (2005) models, assuming a star formation history (SFH) of the form $\psi(t) \propto \exp (-t / \tau)$ and leaving age $(t)$, the $e$-folding time $(\tau)$, the amount of dust attenuation $\left(A_{V}\right)$, and metallicity $(Z)$ as free parameters (see note to Table 1 ). We assume the Calzetti et al. (2000) attenuation curve, which we implement as a uniform screen. We adopt the Salpeter (1955) initial mass function (IMF), as this IMF is available for both SPS models. Compared to a Kroupa (2001) or Chabrier (2003) IMF our choice will primarily affect the mass-to-light ratio and has little impact on other stellar population properties. For each bin, we determine the effective filter curve, by adding the deredshifted filter curves of all included data points. In most cases, this is a variety of broadband and medium-band filters with different observed wavelengths, as our sample spans a large range in redshift.

We start by fitting the full wavelength range. The best fits for Bruzual \& Charlot (2003) and Maraston (2005) are represented by the orange and purple fits in Figure 3. respectively. The Bruzual \& Charlot (2003) models yield an acceptable fit to the data, while the Maraston (2005) models do not fit the full wavelength range simultaneously. The best-fit stellar population properties are broadly consistent, with a slightly lower value for stellar mass $\left(M_{*}\right)$ and $A_{V}$ for the Maraston (2005) models (Table 1). We have explored other SFHs as well, such as a delayed exponential $\mathrm{SFH}(\psi(t) \sim t \exp (-t / \tau))$, a truncated $\mathrm{SFH}$ preceded by a constant star formation rate (SFR, with $\left.\tau=t_{\text {stop }}-t_{\text {start }}\right)$, a truncated SFH preceded by an exponentially increasing SFR, and a two-component population, but none provide a significantly better correspondence between the Maraston (2005) models and the composite SED. The SFH used to generate the model SEDs in Figure 3 produces a sharply peaked spectrum; adding an older or dusty star-forming population would only broaden the model SED, which would then produce an even larger discrepancy between the Maraston (2005) models and the data.

To better understand the discrepancies between the models in the rest-frame near-infrared, we repeat the fitting, but now restricting the wavelength range to $\lambda<$ $6000 \AA$. The Bruzual \& Charlot (2003) and Maraston (2005) models, as represented by the red and blue curves, respectively, fit this wavelength range nearly equally well and yield consistent values for $M_{*}, t, \tau$, and $A_{V}$ (Table 11). However, while Bruzual \& Charlot (2003) also fit the full wavelength coverage, the Maraston (2005) models overpredict the rest-frame near-infrared flux.

\subsection{Tests and Comparison to other Studies}

As a check on our procedure, we stack the best fits to the individual SEDs for both models. The stacks are similar to the purple and orange curves in Figure 3. Along the same lines, we assess the qualities of the individual fits. All galaxies favor the Bruzual \& Charlot (2003) models, with median values for $\chi_{\mathrm{BC} 03}^{2}$ and $\chi_{\mathrm{M} 05}^{2}$ of 0.91 and 4.3 , respectively.

Whereas both the Bruzual \& Charlot (2003) and Maraston (2005) SPS models can reproduce the $B_{\mathrm{m}}-V_{\mathrm{m}}$ and $U_{\mathrm{m}}-B_{\mathrm{m}}$ colors in the selection box, the exact colors at fixed age and star formation timescale are slightly different. In order to test whether these small differences cause a bias that favors a particular SPS model, we split the post-starburst galaxy sample at $B_{\mathrm{m}}-V_{\mathrm{m}}=0.55$, and repeated the analysis for both sub-samples. Both SEDs are significantly better fit by the Bruzual \& Charlot (2003) models (with similar fit qualities as for the full composite spectrum), where the redder sample is older by $\sim 0.1$ dex. Additionally, we repeat the analysis for a redder boundary $B_{\mathrm{m}}-V_{\mathrm{m}}<0.6$, yielding a sample of $\sim 110$ galaxies. The composite spectrum is better fit by a slightly older stellar population, but still strongly favors the Bruzual \& Charlot (2003) models above the 


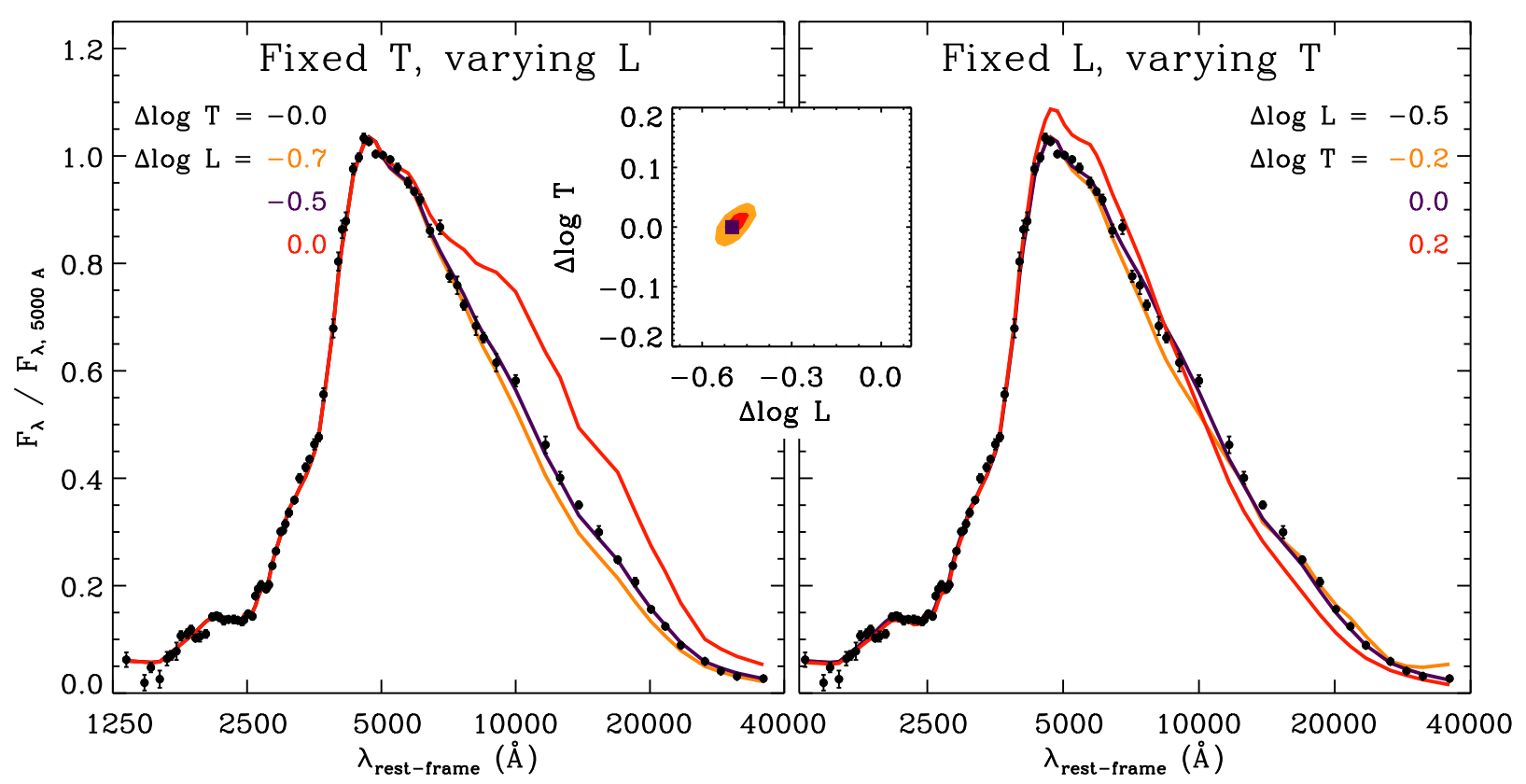

FIG. 4.- Comparison of the composite post-starburst galaxy spectrum with the FSPS models by Conrov et al. (2009), for which the temperature and bolometric luminosity of the TP-AGB stars can be modified with respect to the Padova stellar evolution models $(\Delta \log T$ and $\Delta \log L$, respectively). The left panel illustrates the influence of varying the luminosity, while keeping the temperature fixed to the best-fit value. In the right panel, the luminosity is fixed to the best-fit value, but the temperature varies. In both cases, the best fit is shown in purple. For both panels, $t, \tau$, and $A_{V}$ are fixed as well. The inset shows the best-fit $\Delta \log T$ and $\Delta \log L$ (purple square), and its corresponding $68 \%$ and $95 \%$ confidence intervals.

Maraston (2005). Altogether, this confirms our unbiased selection.

MacArthur et al. (2010) compare the near-infrared fluxes of two spiral galaxies to predictions from long-slit spectra, reporting better consistency with the Maraston (2005) models than with Bruzual \& Charlot (2003). However, the interpretation of their results is complicated by the presence of a significantly older stellar population, which contributes $26 \%-67 \%$ to the total light, and the presence of dust, which may mimic the effects of a TP-AGB star population. Nonetheless, it is possible that the Maraston (2005) model indeed provides a better description for the SED shape during the evolution phase of these spiral galaxies.

Maraston et al. (2006, 2007) use the broadband photometry of seven spectroscopically confirmed galaxies to assess the fit quality of different SPS models and find no overall preference for a particular SPS model. Only one galaxy (ID 3650) of this sample would have entered our selection (see Figure 2). We have re-fitted this galaxy using the same method as for the composite spectrum, and - broadly consistent with Maraston et al. (2006) we neither find a strong preference for a particular SPS model (see Table 1). Thus, the differences in fitting techniques do not account for the large discrepancy in $\chi^{2}$ value for the composite spectrum. Moreover, as several galaxies in our sample, in particular at higher redshift, are almost equally well fit by the Maraston (2005) models, our study is not in disagreement with Maraston et al. $(2006)$.

This redshift dependence may be caused by the fact that the metallicity and dust content at fixed stellar mass evolve with redshift (e.g., Erb et al. 2006; Maiolino et al. 2008), and thus it may be possible that the contribution from TP-AGB stars also changes with time. We test this by splitting our sample at $z=1.56$ and repeating the analyses for both sub-samples. Although, for both samples the Bruzual \& Charlot (2003) models provide significantly better fits, the relative fit quality of the Maraston (2005) models versus Bruzual \& Charlot (2003) increases with redshift. Future work might be able to separate these trends and constrain TP-AGB models as a function of stellar mass and metallicity.

\subsection{Utilizing a Flexible SPS model}

The difference in fit quality between the Bruzual \& Charlot (2003) and Maraston (2005) models for the composite post-starburst galaxy spectrum reflects the different treatments of the TP-AGB phase. Our results suggest that the treatment by Bruzual \& Charlot (2003) is more appropriate for our post-starburst galaxy sample than that of the Maraston (2005) models. We use the flexible SPS10 (FSPS) models by Conroy et al. $(2009,2010)$ to obtain a more quantitative constraint on the TP-AGB phase, as FSPS allows the modification of the bolometric luminosity and effective temperature of the TP-AGB stars.

We start with the best-fit FSPS model to the composite post-starburst galaxy spectrum, restricting the wavelength region to $\lambda<6000 \AA$ and assuming the latest default Padova TP-AGB models Girardi et al. 2000; Marigo \& Girardi 2007; Marigo et al. 2008). Similar to the other models, this is a stellar population with $\log (t / \mathrm{yr})=9.04, \log (\tau / \mathrm{yr})=8.1$, and an $A_{V}$ of $0.4 \mathrm{mag}$ (for solar metallicity). Next, we fix the age, star forma-

10 These models can be downloaded at www.cfa.harvard.edu/ cconroy/FSPS.html. 
tion timescale and dust content of the stellar population and vary both the effective temperature and bolometric luminosity of the TP-AGB stars, quantified as shifts with respect to the Padova evolutionary tracks, $\Delta \log T$ and $\Delta \log L$, respectively. Thus, we ignore any potential degeneracies between $\Delta \log T, \Delta \log L$, and other stellar population properties (Conroy et al. 2009).

Figure 4 illustrates the influence of both parameters on the SED and shows their $\chi^{2}$ contours. The best fit has a reduced $\chi^{2}$ value of 0.75 , thus similar to Bruzual \& Charlot (2003). The composite poststarburst galaxy spectrum suggests that the predicted effective temperatures of TP-AGB stars in the Padova models are consistent with our observations, but the overall luminosity is $\sim 0.5$ dex lower.

\section{CONCLUSIONS}

In this Letter, we define a photometrically selected sample of 62 post-starburst galaxies from the NMBS and use their composite SED to obtain new constraints on the SED shape during the time that the TP-AGB stars are thought to be most dominant. The SED is well fit by the Bruzual \& Charlot (2003) SPS models, while the Maraston (2005) models do not reproduce the rest-frame optical and near-infrared parts of the SED simultaneously, implying that these models give too much weight to TP-AGB stars. This has previously been found by Conroy \& Gunn (2010) using post-starburst galaxies in the SDSS.

The high-resolution photometric sampling of the NMBS allows us to derive quantitative constraints on the luminosity in the TP-AGB phase. Using the FSPS models by Conroy et al. (2009), we find that the bolometric luminosity of TP-AGB stars is a factor of $\sim 3$ lower than predicted by the latest Padova TP-AGB mod- els, when assuming solar metallicity. However, an independent abundance measurement is needed to break the degeneracy between metallicity and the TP-AGB phase parameters.

The significant reduction in the bolometric luminosity of TP-AGB stars that is required to fit the observed post-starburst SED reflects one or more failures of the current generation of SPS models. This reduction can be physically achieved in the models by reducing TPAGB lifetimes, reducing bolometric luminosities of stars in the TP-AGB phase, and/or embedding a significant fraction of TP-AGB stars within optically thick circumstellar dust shells. These different explanations may be disentangled by appealing to mid-IR data (see, e.g., Kelson \& Holden 2010; Salim et al. 2009).

Taking our results at face value, we infer that stellar masses and other stellar population parameters derived using the Maraston (2005) might be biased for certain evolutionary phases. We stress that our findings do not imply that the Bruzual \& Charlot (2003) models are best for all galaxies or for all evolutionary phases. Using similar techniques as presented in this Letter, in combination with flexible SPS models, which allow unrestricted modification of uncertain evolution phases and input parameters (such as metallicity), it will be possible to substantially reduce many of the major uncertainties plaguing all SPS models.

We thank the referee for a very constructive report, Jenny Greene, James Gunn, and Scott Trager for useful discussions, and the COSMOS and AEGIS teams for the release of high-quality multi-wavelength data sets to the community.

\section{REFERENCES}

Brammer, G., van Dokkum, P. G., \& Coppi, P. 2008, ApJ, 686, 1503

Brammer, G., et al. 2009, ApJ, 706, L173

Bruzual, G. \& Charlot, S. 2003, MNRAS, 344, 1000

Calzetti, D., Armus, L., Bohlin, R. C., Kinney, A. L., Koornheef, J., \& Storchi-Bergmann, T. 2000, ApJ, 533, 682

Chabrier, G. 2003, PASP, 115, 763

Conroy, C., \& Gunn, J. E. 2010, ApJ, 712, 833

Conroy, C., Gunn, J. E., \& White, M. 2009, ApJ, 699, 486

Conroy, C., White, M., \& Gunn, J. E. 2010, ApJ, 708, 58

Davis, M., et al. 2007, ApJ, 660, L1

Eminian, C., Kauffmann, G., Charlot, S., Wild, V., Bruzual, G., Rettura, A., \& Loveday, J. 2008, MNRAS, 384, 930

Erb, D. K., Shapley, A. E., Pettini, M., Steidel, C. C., Reddy, N. A., \& Adelberger, K. L. 2006, ApJ, 644, 813

Förster Schreiber, N. M., et al. 2004, ApJ, 616, 40

Girardi, L., Bressan, A., Bertelli, G., \& Chiosi, C. 2000, A\&AS, 141,371

Gonzalez, V., Labbé, I., Bouwens, R. J., Illingworth, G., Franx, M., Kriek, M., \& Brammer, G. B. 2010, ApJ, 713, 115

Goto, T. 2007, MNRAS, 387, 187

Kannappan, S. J., \& Gawiser, E. 2007, ApJ, 657, L5

Kelson, D. D., \& Holden, B. P. 2010, ApJ, 713, L28

Kriek, M., van Dokkum, P. G., Franx, M., Illingworth, G. D., \& Magee, D. K. 2009a, ApJ, 705, L71

Kriek, M., van Dokkum, P. G., Labbé, I., Franx, M., Illingworth, G. D., Marchesini, D., \& Quadri, R. F. 2009b, ApJ, 700, 221

Kroupa, P. 2001, MNRAS, 322, 231

Labbé, I. et al. 2005, ApJ, 624, L81

Labbé, I. et al. 2010, ApJ, 708, L26

Leitherer, C., et al. 1999, ApJS, 123, 3
MacArthur, L. A., McDonald, M., Courteau, S., \& González, J. J. 2010, ApJ, 718, 768

Maiolino, R., et al. 2008, A\&A, 488, 463

Maraston, C. 2005, MNRAS, 362, 799

Maraston, C., Strömbäck, G., Thomas, D., Wake, D. A., \& Nichol, R. C. 2009, ApJ, 394, L107

Maraston, C., et al. 2006, ApJ, 652, 85

Maraston, C., et al. 2007, ApJ, 656, 1241

Marchesini, D., van Dokkum, P. G., Förster Schreiber, N. M., Franx, M., Labbé, I., \& Wuyts, S. 2009, ApJ, 701, 1765

Marigo, P., \& Girardi, L. 2007, A\&A, 469, 239

Marigo, P., Girardi, L., Bressan, A., Groenewegen, M. A. T., Silva, L., \& Granato, G. L. 2008, A\&A, 482, 883

Miller, N. A., \& Owen, F. N. 2001, ApJ, 554, L25

Muzzin, A., Marchesini, D., van Dokkum, P. G., Labbé, I., Kriek, M., \& Franx, M. 2009, ApJ, 701, 1839

Papovich, C., Dickinson, M., \& Ferguson, H. C. 2001, ApJ, 559, 620

Quintero, A. D., et al. 2004, ApJ, 602, 190

Rudnick, G., et al. 2006, ApJ, 650, 624

Salim, S., et al. 2009, ApJ, 700, 161

Salpeter, E. E. 1955, ApJ, 121, 161

Scoville, N., et al. 2007, ApJS, 172, 1

Shapley, A. E., et al. 2005, ApJ, 626, 698

Stark, D. P., Bunker, A. J., Ellis, R. S., Eyles, L. P., \& Lacy, M. 2007, ApJ, 659, 84

Tinsley, B. M. \& Gunn, J. E. 1976, ApJ, 203, 52

van der Wel, A., Franx, M., Wuyts, S., van Dokkum, P. G.,

Huang, J., Rix, H.-W., \& Illingworth, G. D. 2006, ApJ, 652, 97 van Dokkum, P. G., et al. 2009, PASP, 121, 2

van Dokkum, P. G., et al. 2010, ApJ, 709, 1018

Whitaker, K. E., et al. 2010, ApJ, 719, 1715 
Wuyts, S., et al. 2007, ApJ, 655, 51 